\title{
REVIEW
}

\section{Pharmacotherapy for Neuropathic Pain: A Review}

Diego Fornasari

Received: September 19, 2017

(c) The Author(s) 2017. This article is an open access publication

\begin{abstract}
Neuropathic pain, comprising a range of heterogeneous conditions, is often severe and difficult to manage, and this may result in a chronic condition that negatively affects the overall functioning and quality of life in patients. The pharmacotherapy of neuropathic pain is challenging and for many patients effective treatment is lacking; therefore, evidence-based recommendations are essential. Currently, there is general agreement on which drugs are appropriate for the first-line treatment of neuropathic pain, whereas debate continues regarding second- and third-line treatments. First-line drugs for neuropathic pain include antidepressants (tricyclic antidepressants and serotonin-noradrenaline reuptake inhibitors) and anticonvulsants acting at calcium channels (pregabalin and gabapentin). Second- and third-line drugs for neuropathic pain include topical lidocaine and opioids. Although efficacious in the treatment of neuropathic pain, opioids are not considered to be a first choice because of adverse drug reactions and, more recently, because of concerns about abuse, diversion, and addiction. A clear
\end{abstract}

Enhanced content To view enhanced content for this article go to http://www.medengine.com/Redeem/ 20DCF060253B492B.

D. Fornasari $(\bowtie)$

Department of Medical Biotechnology and

Translational Medicine, Università degli Studi di

Milano, Milan, Italy

e-mail: diego.fornasari@unimi.it understanding of the mechanism of action of currently available drugs is an essential step towards an effective clinical approach that aims to tailor therapies both to the specific neuropathic disease and to the needs of an individual patient. This review provides an overview of current drugs available for the treatment of neuropathic pain with an emphasis on their mechanism of action.

Funding: Pfizer, Italy.

Keywords: Anticonvulsants; Antidepressants; Gabapentin; Mechanism of action; Neuropathic pain; Opioids; Pregabalin; Serotonin-noradrenaline reuptake inhibitors; Tricyclic antidepressants

\section{INTRODUCTION}

Neuropathic pain comprises a wide range of heterogeneous conditions caused by lesions or diseases of the somatosensory system, either at the peripheral or at the central level. Neuropathic pain is often severe and difficult to manage, resulting in a chronic condition that negatively affects the overall functioning and quality of life in patients and leads to a high economic burden for the individual and society. The use of effective therapies to control pain and its consequences is, therefore, of primary importance. Many different clinical practice guidelines have been published in the last 
15 years to help clinicians choose appropriate drugs for the management of neuropathic pain [1]. When considering the inherent limitations that are introduced when large amounts of homogenous studies are combined [2, 3], emphasis needs to be placed on guidelines that incorporate practical recommendations made on clinically based evidence. The overwhelming majority of these guidelines are the result of an evidence-based approach, sometimes accompanied by a consensus statement. There is currently general agreement on which drugs are appropriate for first-line treatment of neuropathic pain, whereas the debate regarding second- and third-line drugs is still open, especially concerning weak and strong opioids. Although efficacious in the treatment of neuropathic pain, opioids are not considered to be a first choice because of adverse drug reactions and, more recently, because of concerns about abuse, diversion, and addiction [4]. First-line drugs for neuropathic pain include antidepressants (tricyclic antidepressants [TCA] and serotonin-noradrenaline reuptake inhibitors [SNRI]) and anticonvulsants acting at calcium channels (pregabalin and gabapentin) [5].

\section{Compliance with Ethics Guidelines}

This article is based on previously conducted studies and does not involve any new studies of human or animal subjects performed by the author.

\section{FIRST-LINE DRUGS FOR NEUROPATHIC PAIN}

\section{Antidepressants}

Antidepressants are among the oldest drugs used for the treatment of neuropathic pain and have been the subject of many randomized controlled trials. They originally came to be used in the treatment of chronic pain, and in particular neuropathic pain, because some of the patients suffering from chronic pain are also depressed, and these drugs relieve pain as well as depression. However, an independent analgesic action has been reported for TCAs since the 1960s. Pain relief has since been described in depressed and non-depressed patients with chronic pain. The relief can be more rapid in some patients and appears to occur at a lower dose than the antidepressant effect [6]. An early concept of the mechanism of antidepressant analgesia was that these drugs are capable of potentiating the activity of the descending inhibitory pathways extending from the brain stem to the dorsal horn of the spinal cord, mainly by inhibiting the reuptake of serotonin and noradrenaline that descending fibers release into the spinal synapses between nociceptors (or first-order neurons) and the spinothalamic neurons (or second-order neurons). At this level, neurotransmitters can inhibit synaptic transmission between first- and second-order neurons directly, by binding to membrane receptors expressed on the surface of these neurons, as in the case of noradrenaline that binds alpha- 2 adrenergic receptors. Alternatively, they can activate interneurons that in turn release inhibitory substances such as endogenous opioids or gamma-aminobutyric acid (GABA), as in the case of serotonin at its metabotropic receptors or noradrenaline at alpha-1 adrenergic receptors [7]. Recently, some studies highlighted a possible peripheral mechanism for the action of antidepressants. Bohren and colleagues examined the effect of noradrenergic lesions at different levels of the nervous system and concluded that the analgesic properties of long-term nortriptyline rely on the peripheral noradrenergic system (whereby the sympathetic fibers sprouting in the dorsal root ganglia that accompany the nerve injury are the source of noradrenaline) [8].

The most effective antidepressants for neuropathic pain appear to be TCAs, in particular desipramine, amitriptyline and its metabolite, nortriptyline, and imipramine. TCAs are relatively "dirty drugs" that affect multiple targets and have pleiotropic effects. This lack of selectivity contributes to their efficacy. For instance, it has been shown that amitriptyline can act as a local anesthetic by blocking voltage-gated sodium channels [9]. Antidepressants may have additional mechanisms of action by modulating the immune system, which is heavily involved 
in neuropathic pain. Moreover, TCAs may directly interfere with central sensitization by blocking NMDA receptors in the spinal cord (see Kremer et al. [10] for a review on the pharmacodynamics of antidepressants). TCAs have been proven to be efficacious in several neuropathic conditions, including painful polyneuropathy, post-herpetic neuralgia, peripheral nerve injury, and painful diabetic neuropathy [5].

On the other hand, the multiple actions of TCAs are also responsible for many adverse drug reactions that limit their use. In particular, anticholinergic effects are a major concern because of the risk of cardiotoxicity, limiting the dosage to less than $100 \mathrm{mg} /$ day; they also include dry mouth, orthostatic hypotension, constipation, and urinary retention. In order to overcome these problems, selective SNRIs, in particular duloxetine, have been introduced in the treatment of neuropathic pain. SNRIs have been proven to be efficacious in several neuropathic conditions including painful polyneuropathy, post-herpetic neuralgia, painful diabetic neuropathy, and low back pain [5].

Duloxetine has shown consistent efficacy in painful diabetic neuropathy and low back pain $[11,12]$. Dosing of duloxetine is simple with $60 \mathrm{mg}$ once or twice daily appearing to be equally effective. Nausea is the most common adverse effect of duloxetine, which appears to be reduced by lowering the dosage to $30 \mathrm{mg}$ once daily for 1 week before increasing to $60 \mathrm{mg}$ once daily.

\section{Anticonvulsants Acting at Voltage-Gated Calcium Channels}

Pregabalin and gabapentin are both derived from GABA, but they have no effect on the GABAergic system. Their mechanism of action includes binding to the alpha-2/delta-1 subunit of the voltage-gated calcium channels in several areas of the central nervous system (CNS) and spinal cord in which these channels are expressed, and this is sufficient to explain their analgesic, anxiolytic, and anticonvulsant pharmacological properties [13]. Voltage-gated calcium channels are localized on presynaptic terminals, where they control neurotransmitter release. Being voltage-sensitive, they open in response to action potentials arising from the periphery and allow the influx of calcium ions, which is essential for the fusion of synaptic vesicles and release of neurotransmitters into the synaptic cleft (Fig. 1). This is the general mechanism by which these channels are involved in neurotransmitter release in the spinal cord and in various areas of the CNS.

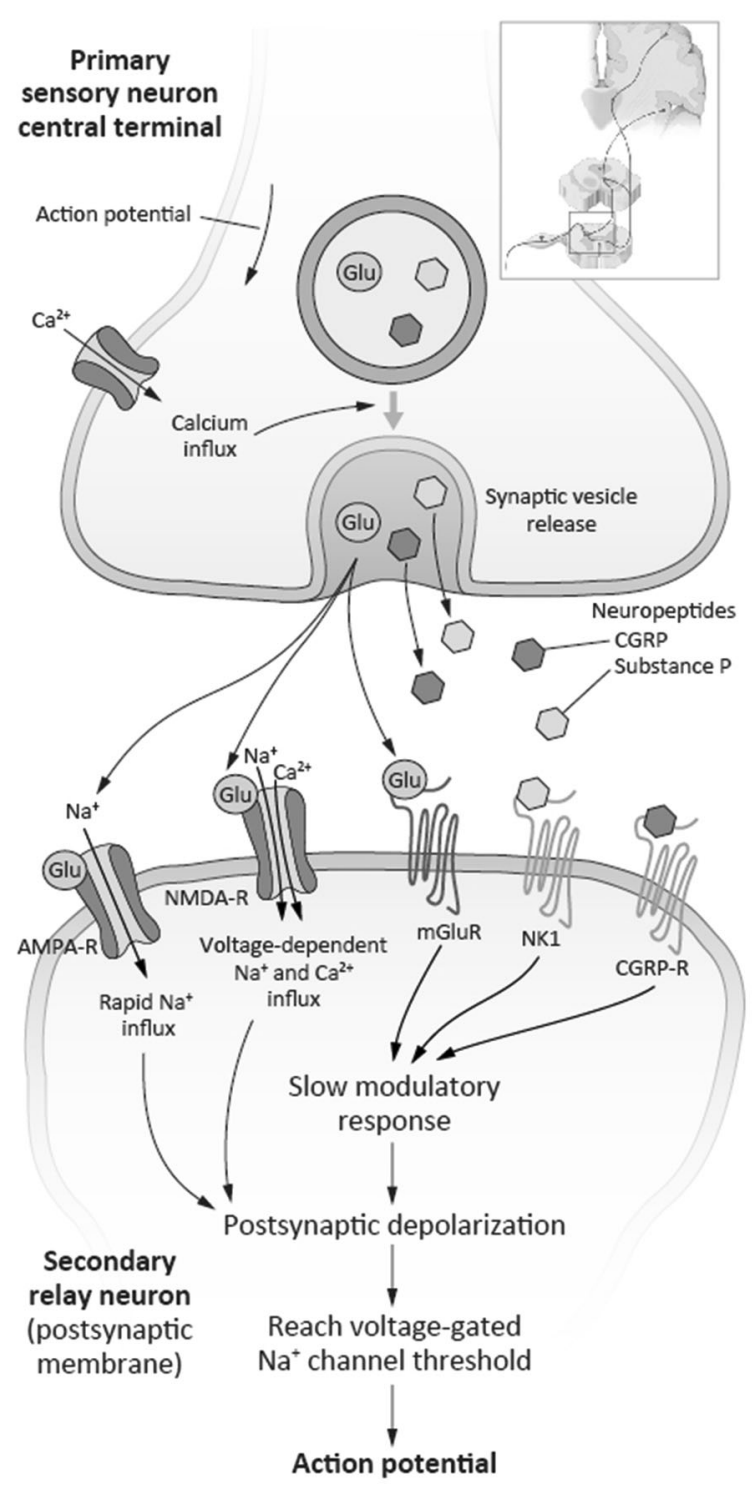

Fig. 1 Spinal synapse between the nociceptor and the spinothalamic neuron Reproduced with permission from [30] 
Voltage-gated calcium channels are comprised of different subunits: the alpha subunit is responsible for the formation of the pore through which calcium ions enter into the cell, whereas the alpha-2/delta-1, beta, and gamma are accessory subunits. The alpha-2/delta-1 subunit is responsible for the trafficking, localization, and stabilization of the channel in the plasma membrane. Interestingly, it has been demonstrated that the alpha-2/delta-1 subunit binds at one site to the alpha- 1 subunit and at another site to thrombospondin, a protein of the extracellular matrix which is produced by activated astrocytes. Since in experimental models of neuropathic pain thrombospondin appears to be upregulated, it is possible to speculate that the activation of astrocytes in the spinal cord as a consequence of nerve injury and the abnormal secretion of thrombospondin promote the stabilization of voltage-gated calcium channels at the presynaptic terminal (Fig. 2). Indeed, it has been shown that the number of voltage-gated calcium channels increases in neuropathic pain conditions and may sustain an aberrant neurotransmission in the spinal cord. Therefore, it is likely that gabapentinoids, by binding to the alpha-2/ delta-1 subunit, destabilize the macromolecular complex that keeps the calcium channel on the surface of the presynaptic terminal, promoting its internalization [13]. Thus, gabapentinoids have no direct effect on the currents, but

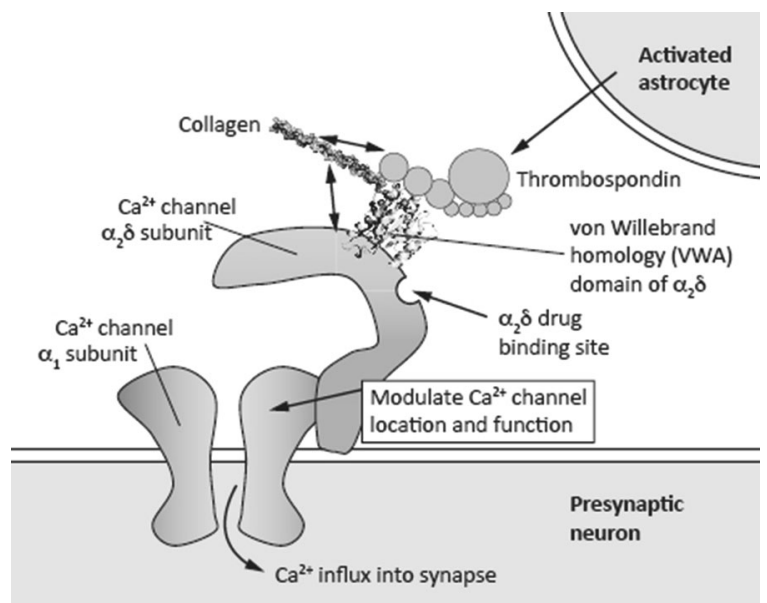

Fig. 2 Mechanism of action of pregabalin and gabapentin Reproduced with permission from [13] instead influence the number of available calcium channels in the plasma membrane.

Brainstem structures, from which descending modulatory fibers originate, may be a key target of the analgesic action of gabapentinoids, because alpha-2/delta-1 expression is very high in these areas. Microinjection of gabapentin into the locus coeruleus (LC) reduced neuropathic pain behaviors in rats, whilst different responses in the LC and spinal dorsal horn were evoked by gabapentin administered intravenously in rats with and without L5-L6 spinal nerve ligation [14]. From this, it was concluded that gabapentin reduced presynaptic GABA release in the LC but not in the spinal dorsal horn. Studies using animal models have suggested that presynaptic release of GABA in the LC is reduced by alpha-2/delta-1 ligands and that this restores descending noradrenergic inhibition after nerve injury [15]. Therefore, the antinociceptive activities of pregabalin and gabapentin are also associated with descending noradrenergic and serotonergic activity, through which pain transmission in the spinal cord is modulated. Although the general mechanisms of action of pregabalin and gabapentin are similar, important differences exist in terms of pharmacodynamics [16]. Pregabalin has greater binding affinity for the alpha-2/delta-1 subunit, and therefore its analgesic potency in neuropathic pain is higher compared with gabapentin, thus justifying the utility of converting from gabapentin to pregabalin if the first drug is not effective enough. Major differences also exist in terms of pharmacokinetics, especially absorption. The system-L protein family [L-type amino acid transporters (LAT)] enables the transport of large neutral amino acids, including phenylalanine, leucine, isoleucine, and valine; intestinal absorption of gabapentin and pregabalin is also facilitated by this protein family. Results from preclinical studies suggest that gabapentin is transported exclusively by the LAT1 transporter. This results in dose-limited absorption, which may be due to saturation of the facilitated transport process. An additional pathway also appears to mediate the absorption of pregabalin, resulting in a high level of absorption into the bloodstream [16]. This obviously reflects on bioavailability, 
which for gabapentin decreases at higher dosage.

Other pharmacokinetic parameters are similar for the two drugs and deserve to be discussed because of their impact in clinical practice. Both drugs do not undergo metabolism by phase I or phase II enzymes and are excreted unmodified by the kidneys. This implies that the two drugs are not prone to pharmacokinetic drug-drug interactions and, specifically, are not substrates of the cytochrome P450 (CYP) system, which is involved in the metabolism of many other drugs. This is of significant clinical value, because the two drugs can be safely used in comorbid patients on pharmacological polytherapy. For the same reason gabapentin and pregabalin can be safely used in combination with other analgesic drugs used in the treatment of neuropathic pain. Gabapentinoids have been proven to be efficacious in several neuropathic conditions such as post-herpetic neuralgia, painful diabetic neuropathy, painful polyneuropathy, and low back pain.

In general, pregabalin and gabapentin are well tolerated. The most commonly reported adverse effect of pregabalin is dizziness, followed by somnolence, dry mouth, edema, and blurred vision, with treatment discontinuation due to somnolence occurring in $4 \%$ of patients. For gabapentin, dizziness and somnolence occur in more than $20 \%$ of patients and are the most commonly reported adverse effects; other adverse effects include confusion and peripheral edema. For both drugs, adverse effects are dose-dependent and reversible [16].

\section{SECOND- AND THIRD-LINE DRUGS FOR NEUROPATHIC PAIN}

\section{Topical Lidocaine}

Lidocaine, in the form of 5\% patches, was efficacious and had an excellent tolerability profile in randomized controlled trials of patients with post-herpetic neuralgia and allodynia, as well as in patients with allodynia due to neuropathic pain [17]. Lidocaine blocks voltage-gated sodium channels that are expressed by nerve fibers, which are responsible for the propagation of action potentials. The number, localization, subtype expression, and activity of these channels are altered in different forms of neuropathic pain [18]. Because topical lidocaine can penetrate no deeper than $8-10 \mathrm{~mm}$, it is therefore indicated in well-localized neuropathic pain. Its efficacy has been documented in different types of localized neuropathic pain, including post-herpetic neuralgia, painful diabetic neuropathy, post-surgical and post-traumatic pain related to incision of the skin [19]. The most common adverse effects of lidocaine are mild local reactions due to its topical application. Lidocaine's lack of systemic absorption and of drug interactions can be particularly beneficial in older patients [19].

\section{Opioids}

Strong opioids, such as morphine, oxycodone, and hydromorphone, and weak opioids, such as tramadol, are efficacious when compared with other drugs used for neuropathic pain and are similar to antidepressants in terms of the numbers needed to treat [5]. Nevertheless, they have always been considered second-line drugs [1], and more recently third-line drugs [5], due to adverse drug reactions and concerns about abuse, diversion, and addiction. Tapentadol represents a new class of dual opioid analgesics, combining a less potent agonistic activity at mu-opioid receptors with inhibition of noradrenaline uptake, and exploiting the synergy between the two mechanisms. The innovative pharmacodynamics and a favorable pharmacokinetic profile make tapentadol a unique opioid analgesic. However, the paucity of available studies prevented tapentadol from being included in the most recent systematic review and meta-analysis on neuropathic pain [20-22]. For this reason it will not be further discussed in this review.

The analgesic effect of opioids is due to their action in the brain, brainstem, spinal cord, and, under certain circumstances, on peripheral terminals of primary afferent neurons. All endogenous opioid peptides, including $\beta$-endorphin, enkephalins, and dynorphins, bind to seven transmembrane $G$ protein-coupled 
receptors, which are divided into three classes: $\mathrm{mu}$, delta, and kappa receptors. Opioid receptors are coupled to inhibitor $G$ proteins, with receptor activation inhibiting the adenylate cyclase as well as the intracellular production of cAMP. However, the coupling of opioid receptors to calcium and potassium channels is thought to be a central mechanism of analgesia production by both endogenous and exogenous opioids.

In the dorsal horn of the spinal cord, mu receptors make up the majority of opioid receptors with over $70 \%$ of the presynaptic location occurring at the central terminals of nociceptors (C and A delta fibers). Opioid receptors located postsynaptically on dendrites of second-order spinothalamic neurons and on interneurons make up the remaining 30\%. Interneurons are predominately responsible for the release of endogenous opioids beta-enkephalin and endorphins, which act on mu receptors in the dorsal horn of the spinal cord. The activation of interneurons is dependent on the activity of descending pathways or, in a direct manner, by descending fibers. Inhibition of calcium ion channels is caused by the activation of presynaptic mu receptors, thus preventing neurotransmitter release. Activation of potassium ion channels is caused by the activation of postsynaptic mu receptors resulting in the efflux of potassium ions and hyperpolarization of the projecting cell. Therefore, stimulation of mu-opioid receptors in the spinal cord is an effective mechanism of blocking synaptic transmission, which restricts the number of nociceptive stimuli reaching the thalamus and cortex, in which a conscious perception of pain occurs [23].

In spite of their efficacy, the role of opioids in the long-term treatment of nonmalignant pain is controversial for a number of reasons, including concerns over tolerability, possible development of tolerance to the analgesic effect, and the risk of addiction [24]. A systematic review of randomized controlled trials of oral opioids for chronic nonmalignant pain indicated that approximately $50 \%$ of patients experienced an adverse event with opioids and more than $20 \%$ discontinued treatment because of adverse events [25]. A more recent Cochrane review of long-term opioid management of chronic non-cancer pain reported a rate of discontinuation due to adverse events of $22.9 \%$ for oral opioids and $12.1 \%$ for transdermal opioids [26]. There was a significant difference in discontinuation rates for orally administered weak (11.4\%) compared with strong (34.1\%) opioids. However, many studies in this Cochrane review specified that most adverse events were minor and some of them diminished over time [26]. The most frequent adverse drug reactions to opioid therapy are nausea and vomiting (tend to diminish with increasing tolerance), constipation (remains a constant problem), pruritus, respiratory depression (very uncommon), dry mouth, urinary retention, drowsiness, and cognitive impairment. Drowsiness and cognitive impairment should be considered, along with constipation, the most serious adverse drug reactions to opioids, because they can seriously affect the patients' quality of life. Over the last few years, addiction, diversion, and abuse have become the subject of a worldwide debate that started in the USA in response to the persistent increase of deaths due to unintentional overdose. A detailed discussion of this issue is beyond the scope of this review. Although the risk of addiction is inherent to opioids, its relevance in patients suffering from chronic pain, who take these drugs for pain relief, remains to be elucidated. It is likely that people with a previous experience of drug abuse or people with psychiatric diseases, such as depression or schizophrenia, are at higher risk of developing addiction after taking opioids. In other patients who take opioids for pain relief, the risk is probably very close to that in the general population [27]. Nevertheless, the use of appropriate tools to identify at-risk patients prior to initiating treatment with opioids, constant vigilance on the behavior associated with opioid assumption, and frequent re-evaluation of the balance between risks and benefits of long-term opioid therapies should become a normal attitude among physicians [27].

\section{Other Drugs}

A number of additional drugs have shown efficacy in the treatment of neuropathic pain; 
however, these were either single randomized controlled trials or their efficacy was inconsistent across multiple randomized controlled trials. These drugs represent the third or even fourth line of treatment options for neuropathic pain and include specific antidepressants (e.g., bupropion) and antiepileptic drugs (carbamazepine, lamotrigine), and topical low-concentration capsaicin. In general, these drugs should be reserved for patients who are unable to tolerate or who fail to respond to first- and second-line medications.

\section{CONCLUSION}

The pharmacotherapy of neuropathic pain is challenging and for many patients effective treatment is lacking. There are several reasons for this, including insufficient knowledge on efficacious drugs and their appropriate use in clinical practice. Neuropathic pain is also associated with interference with sleep, depression, and anxiety that, if not properly treated, will negatively influence the responses to analgesic drugs. Therefore, evidence-based recommendations for the pharmacotherapy of neuropathic pain are essential. However, when choosing a drug, physicians should also take into consideration comorbidity, prioritizing the use of those drugs that can satisfy more than one medical need, as for instance gabapentinoids in the case of interference of pain with sleep, or SNRI in the case of associated depression. On the other hand, comorbidities are also a practical problem, because patients taking many medicines are at higher risk of drug-drug interactions that may be responsible for adverse drug reactions or therapeutic failure. Since a large number of drug-drug interactions involve metabolism by the CYP enzymes, drugs which do not undergo metabolism by CYP or do not undergo liver metabolism at all, as in the case of gabapentinoids, are preferable to other drugs. This holds true in the case of combination pharmacotherapy, in which two or more analgesic drugs are co-administered when one is not enough, a therapeutic strategy that seems to have evident advantages [28, 29].
Although new innovative molecules are needed for the successful cure of neuropathic pain, a deeper understanding of the mechanism of action of currently available drugs is an essential step towards an effective clinical approach that tailors therapies both to the specific neuropathic disease and to the needs of an individual patient.

\section{ACKNOWLEDGMENTS}

This supplement has been sponsored by Pfizer, Italy. The article processing charges for this publication were also funded by Pfizer, Italy. Diego Fornasari thanks Georgii Filatov and Dr Melanie Gatt, PhD, of Springer Healthcare Communications, who provided English editing of the manuscript. This medical writing assistance was funded by Pfizer, Italy. The named author meets the International Committee of Medical Journal Editors (ICMJE) criteria for authorship for this manuscript, takes responsibility for the integrity of the work as a whole, and has given final approval to the version to be published.

Disclosures. Diego Fornasari reports receiving honorarium from Pfizer, as well as honoraria or lecture fees from the following pharmaceutical industries: Alfa-Sigma, Angelini, Bayer, Grunenthal, Ibsa, Janssen, Kiowa Kirin, Lundbeck, Molteni, Mundipharma, Recordati, Scharper, Spa, and Teva.

Compliance with Ethics Guidelines. This article is based on previously conducted studies and does not involve any new studies of human or animal subjects performed by the author.

Data Availability. Data sharing is not applicable to this article as no datasets were generated or analyzed during the current study.

Open Access. This article is distributed under the terms of the Creative Commons Attribution-NonCommercial 4.0 International License (http://creativecommons.org/licenses/ by-nc/4.0/), which permits any noncommercial 
use, distribution, and reproduction in any medium, provided you give appropriate credit to the original author(s) and the source, provide a link to the Creative Commons license, and indicate if changes were made.

\section{REFERENCES}

1. Deng Y, Luo L, Hu Y, Fang K, Liu J. Clinical practice guidelines for the management of neuropathic pain: a systematic review. BMC Anesthesiol. 2016;16:12.

2. Ioannidis JP. Why most published research findings are false. PLoS Med. 2005;2:e124.

3. Chalmers I, Bracken MB, Djulbegovic B, et al. How to increase value and reduce waste when research priorities are set. Lancet. 2014;383:156-65.

4. Dowell D, Haegerich TM, Chou R. CDC guideline for prescribing opioids for chronic pain-United States, 2016. MMWR. 2016;65:1-49.

5. Finnerup NB, Attal N, Haroutounian S, et al. Pharmacotherapy for neuropathic pain in adults: a systematic review and meta-analysis. Lancet Neurol. 2015;14:162-73.

6. Hameroff SR, Weiss JL, Lerman JC, et al. Doxepin's effects on chronic pain and depression: a controlled study. J Clin Psychiatry. 1984;45:47-53.

7. Bee L, Dickenson A. Descending modulation of pain. In: Malcangio M, editor. Synaptic plasticity in pain. New York: Springer Verlag; 2009. p. 307-35.

8. Bohren Y, Tessier LH, Megat S, et al. Antidepressants suppress neuropathic pain by a peripheral beta2-adrenoceptor mediated anti-TNFalpha mechanism. Neurobiol Dis. 2013;60:39-50.

9. Sudoh Y, Cahoon EE, Gerner P, Wang GK. Tricyclic antidepressants as long-acting local anesthetics. Pain. 2003;103:49-55.

10. Kremer M, Salvat E, Muller A, Yalcin I, Barrot M. Antidepressants and gabapentinoids in neuropathic pain: mechanistic insights. Neuroscience. 2016;338:183-206.

11. Peltier A, Goutman SA, Callaghan BC. Painful diabetic neuropathy. BMJ. 2014;348:g1799.

12. King JB, Schauerhamer MB, Bellows BK. A review of the clinical utility of duloxetine in the treatment of diabetic peripheral neuropathic pain. Ther Clin Risk Manag. 2015;11:1163-75.

13. Stahl SM, Porreca F, Taylor CP, et al. The diverse therapeutic actions of pregabalin: is a single mechanism responsible for several pharmacological activities? Trends Pharmacol Sci. 2013;34:332-9.

14. Yoshizumi M, Parker RA, Eisenach JC, Hayashida K. Gabapentin inhibits gamma-amino butyric acid release in the locus coeruleus but not in the spinal dorsal horn after peripheral nerve injury in rats. Anesthesiology. 2012;116:1347-53.

15. Takeuchi Y, Takasu K, Ono H, Tanabe M. Pregabalin, S-(+)-3-isobutylgaba, activates the descending noradrenergic system to alleviate neuropathic pain in the mouse partial sciatic nerve ligation model. Neuropharmacology. 2007;53:842-53.

16. Bockbrader HN, Wesche D, Miller R, et al. A comparison of the pharmacokinetics and pharmacodynamics of pregabalin and gabapentin. Clin Pharmacokinet. 2010;49:661-9.

17. Dworkin RH, O'Connor AB, Audette J, et al. Recommendations for the pharmacological management of neuropathic pain: an overview and literature update. Mayo Clin Proc. 2010;85:S3-14.

18. Cardoso FC, Lewis RJ. Sodium channels and pain: from toxins to therapies. Br J Pharmacol. 2017. https://doi.org/10.1111/bph.13962.

19. Baron R, Allegri M, Correa-Illanes G, et al. The 5\% lidocaine-medicated plaster: its inclusion in international treatment guidelines for treating localized neuropathic pain, and clinical evidence supporting its use. Pain Ther. 2016;5:149-69.

20. Hartrick CT, Rozek RJ. Tapentadol in pain management: a mu-opioid receptor agonist and noradrenaline reuptake inhibitor. CNS Drugs. 2011;25:359-70.

21. Vadivelu N, Kai A, Maslin B, et al. Tapentadol extended release in the management of peripheral diabetic neuropathic pain. Ther Clin Risk Manag. 2015;11:95-105.

22. Coluzzi F, Fornasari D, Pergolizzi J, Romualdi P. From acute to chronic pain: tapentadol in the progressive stages of this disease entity. Eur Rev Med Pharmacol Sci. 2017;21:1672-83.

23. Fornasari D. Pain pharmacology: focus on opioids. Clin Cases Miner Bone Metab. 2014;11:165-8.

24. Labianca R, Sarzi-Puttini P, Zuccaro SM, et al. Adverse effects associated with non-opioid and opioid treatment in patients with chronic pain. Clin Drug Investig. 2012;32(Suppl 1):53-63. 
25. Moore RA, McQuay HJ. Prevalence of opioid adverse events in chronic non-malignant pain: systematic review of randomised trials of oral opioids. Arthritis Res Ther. 2005;7:R1046-51.

26. Noble M, Treadwell JR, Tregear SJ, et al. Long-term opioid management for chronic noncancer pain. Cochrane Database Syst Rev. 2010;CD006605.

27. Leonardi C, Vellucci R, Mammucari M, Fanelli G. Opioid risk addiction in the management of chronic pain in primary care: the addition risk questionnaire. Eur Rev Med Pharmacol Sci. 2015;19:4898-905.
28. Chaparro LE, Wiffen PJ, Moore RA, Gilron I. Combination pharmacotherapy for the treatment of neuropathic pain in adults. Cochrane Database Syst Rev. 2012;008943.

29. Eisenberg E, Suzan E. Drug combinations in the treatment of neuropathic pain. Curr Pain Headache Rep. 2014;18:463.

30. Golan DE, Armstrong EJ, Armstrong AW. Principles of pharmacology. IV ed. Philadelphia: Lippincott Williams \& Wilkins; 2016. 